\title{
Nitrogen and vacancy clusters in $\mathrm{ZnO}$
}

\author{
Filip Tuomisto a) and Christian Rauch \\ Department of Applied Physics, Aalto University, FI-00076 Aalto, Finland \\ Markus R. Wagner \\ ICN2 - Institut Catala de Nanociencia i Nanotecnologia, Campus UAB, 08193 Bellaterra (Barcelona), Spain; and \\ Institute of Solid State Physics, Technical University Berlin, 10623 Berlin, Germany \\ Axel Hoffmann \\ Institute of Solid State Physics, Technical University Berlin, 10623 Berlin, Germany
}

Sebastian Eisermann and Bruno K. Meyer

I. Physics Institute, Justus-Liebig-University Giessen, 35392 Giessen, Germany

Lukasz Kilanski

Institute of Physics, Polish Academy of Sciences, 02-668 Warsaw, Poland

Marianne C. Tarun and Matthew D. McCluskey

Department of Physics and Astronomy, Washington State University, Pullman, Washington, 99164-2814

(Received 26 March 2013; accepted 18 June 2013)

\begin{abstract}
Understanding the interaction of group $\mathrm{V}$ impurities with intrinsic defects in $\mathrm{ZnO}$ is important for developing p-type material. We have studied $\mathrm{N}$-doped $\mathrm{ZnO}$ thin films and $\mathrm{N}$-doped bulk $\mathrm{ZnO}$ crystals, with positron annihilation spectroscopy, in contrast to earlier studies that have concentrated on $\mathrm{N}$-implanted $\mathrm{ZnO}$ crystals. We show that the introduction of $\mathrm{N}$ impurities into $\mathrm{ZnO}$, irrespective of whether it is done during the growth of thin films or bulk crystals or through implantation and subsequent thermal treatments, leads to the formation of stable vacancy clusters and negative ion-type defects. Interestingly, the stability of these vacancy clusters is found almost exclusively for $\mathrm{N}$ introduction, whereas single $\mathrm{Zn}$ vacancy defects or easily removable vacancy clusters are more typically found for $\mathrm{ZnO}$ doped with other impurities.
\end{abstract}

\section{INTRODUCTION}

$\mathrm{ZnO}$ has great potential to be used as a material for optoelectronics components thanks to its suitable bandgap $(\sim 3.4 \mathrm{eV})$ and high excitation binding energy $(\sim 60 \mathrm{meV}){ }^{1-3}$ However, the difficulties encountered when attempting to dope $\mathrm{ZnO}$ p-type have slowed down the device development. One of the more promising group V dopants for obtaining p-type $\mathrm{ZnO}$ is nitrogen, as it should be easily incorporated on the $\mathrm{O}$ sublattice thanks to its similar size. Doping through both implantation ${ }^{4-6}$ and incorporation during growth ${ }^{7-9}$ are intensively investigated.

Positron annihilation spectroscopy is an effective method for the investigation of vacancy-type defects in semiconductors. Positrons implanted into a solid can get trapped in and localize at neutral and negative vacancies due to the missing positive ion core. This results in observable changes in the measurable annihilation characteristics, i.e., the positron lifetime and the momentum distribution of the annihilating positron-electron pair (Doppler broadening). In addition, positrons may have

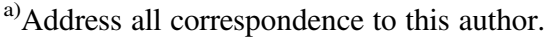
e-mail: filip.tuomisto@aalto.fi

This paper has been selected as an Invited Feature Paper.

DOI: $10.1557 /$ jmr.2013.195
}

shallow hydrogenic states at negative ions, such as acceptor impurities. The annihilation data can be used to determine the vacancy concentration as well as to distinguish between different vacancy types and their chemical environment.

Vacancies in $\mathrm{ZnO}$ have been studied using positron annihilation spectroscopy by many research groups since the late 1980s, most of them dealing with bulk $\mathrm{ZnO}$ crystals and radiation damage. ${ }^{10-12}$ In spite of the considerable research effort especially during the last 10 years, the understanding of many basic characteristics of the defects in $\mathrm{ZnO}$ is still quite limited. In particular, studies of doping effects on the vacancy defects structure are relatively scarce. In this paper, we present results obtained with positron annihilation spectroscopy in $\mathrm{N}$-doped $\mathrm{ZnO}$ thin films and bulk crystals. We compare these new findings to those published earlier on $\mathrm{N}$-implanted $\mathrm{ZnO}$ and discuss the special relationship between $\mathrm{N}$ impurities and vacancy clusters in the $\mathrm{ZnO}$ lattice.

\section{EXPERIMENTAL DETAILS}

\section{A. Samples}

The $\mathrm{ZnO}$ thin films with a thickness of $400-500 \mathrm{~nm}$ were grown by chemical vapor deposition (CVD) using 
metallic zinc, $\mathrm{NO}_{2}$, and $\mathrm{NH}_{3}$ as precursors. The nitrogen concentration was varied by adjusting the dopant flow between 20 and $100 \mathrm{sccm}$ using a constant growth temperature of $350{ }^{\circ} \mathrm{C}$. The low temperature was chosen to push the achievable nitrogen concentration into the $10^{20}$ per $\mathrm{cm}^{3}$ range as the solubility of nitrogen drastically decreases with increasing growth temperature. ${ }^{8}$ All films were grown on nonpolar a-plane substrates since the incorporation of unintentional impurities during the CVD process is reduced by using either a-plane or $c$-plane $\mathrm{Zn}$-face substrates as compared with $\mathrm{O}$-face $\mathrm{ZnO}{ }^{13,14}$ and higher nitrogen doping levels can be achieved. ${ }^{7,8}$

Bulk $\mathrm{ZnO}$ samples were grown by seeded chemical vapor transport (CVT) using ammonia $\left(\mathrm{NH}_{3}\right)$ as a source of nitrogen dopants. ${ }^{15} \mathrm{ZnO}$ and graphite powder were loaded into one end of a fused silica ampoule. A seed crystal $^{16}$ was attached to the deposition end, $\sim 10 \mathrm{~cm}$ from the source powder. The ampoule was evacuated, backfilled with $1 / 2$ atm $\mathrm{NH}_{3}$, and sealed using hydrogen-oxygen torch. The ampoule was placed in a three-zone horizontal furnace that maintains a temperature of $1000{ }^{\circ} \mathrm{C}$ at the source end and $880{ }^{\circ} \mathrm{C}$ at the deposition end.

\section{B. Positron annihilation spectroscopy}

In this work, the positron lifetimes in bulk crystals were measured using a conventional fast-fast coincidence spectrometer with a time resolution of $250 \mathrm{ps}{ }^{17}$ Two identical sample pieces were sandwiched with a $20 \mu \mathrm{Ci}$ positron source $\left({ }^{22} \mathrm{Na}\right.$ deposited on $1.5-\mu \mathrm{m}$ Al foil). Typically, $2 \times 10^{6}$ annihilation events were collected in each positron lifetime spectrum. The lifetime spectrum $n(t)=\Sigma_{i} I_{i} \exp \left(-t / \tau_{i}\right)$ was analyzed as the sum of exponential decay components convoluted with the Gaussian resolution function of the spectrometer after subtracting the constant background and annihilations in the source material (typically a few percent, for details, see Ref. 18) The positron in state $i$ annihilates with a lifetime $\tau_{i}$ and an intensity $I_{i}$. The state in question can be the delocalized state in the lattice or the localized state at a vacancy defect. The increase of the average lifetime $\tau_{\text {ave }}=\Sigma_{i} I_{i} \tau_{i}$ above the bulk lattice lifetime $\tau_{\mathrm{B}}$ shows that vacancy defects are present in the material. This parameter is insensitive to the decomposition procedure, and even a change as small as $1 \mathrm{ps}$ in its value can be reliably measured. In the case of one type of vacancy defect, with specific lifetime $\tau_{\mathrm{V}}$, the decomposition of the lifetime spectrum into two components $\tau_{1}$ and $\tau_{2}$ is straightforward to interpret. The second lifetime component $\tau_{2}=\tau_{\mathrm{V}}$ gives directly the vacancy-specific lifetime and the first lifetime component is $\tau_{1}=\left(\tau_{\mathrm{B}}{ }^{-1}+\kappa_{\mathrm{V}}\right)^{-1}<\tau_{\mathrm{B}}$, where $\tau_{\mathrm{B}}$ is the positron lifetime in the delocalized state in the lattice and $\tau_{\mathrm{V}}$ is the positron trapping rate into the vacancy defects.

The temperature dependence of the average positron lifetime $\tau_{\mathrm{ave}}$ is analyzed with the model of trapping and escape rates of positrons. ${ }^{18}$ In this model, the trapping coefficient $\mu_{\mathrm{V}}$ to a neutral vacancy is independent of temperature and to a negatively charged vacancy, it varies as $T^{-0.5}$. The trapping rate of positrons into the vacancies (concentration $c_{\mathrm{V}}$ ) is $\kappa_{\mathrm{V}}=\mu_{\mathrm{V}} c_{\mathrm{V}}\left(\mu_{\mathrm{V}}=3 \times 10^{15} \mathrm{~cm}^{3} / \mathrm{s}\right.$ for $\mathrm{Zn}$ vacancies in $\mathrm{ZnO}$ at $300 \mathrm{~K}$ ). Positrons can get trapped also at hydrogen-like Rydberg states surrounding negative ion-type defects (shallow traps for positrons). The positron trapping rate at the Rydberg state $\mu_{\mathrm{R}}$ varies also as $T^{-0.5}$, which is the result predicted by the theory of transition from a free state to a bound state in a Coulomb potential. ${ }^{19}$

The positron annihilation characteristics were measured in thin epitaxial films by using a mono-energetic continuous positron beam. As lifetime experiments are not possible with a continuous beam, the Doppler broadening of the annihilation radiation was monitored as a function of the beam energy, providing a depth profile of the signal. The motion of the annihilating electron-positron pair causes a Doppler shift in the annihilation radiation $\Delta E=c p_{\mathrm{L}} / 2$, where $p_{\mathrm{L}}$ is the longitudinal momentum component of the pair in the direction of the annihilation photon emission. This causes the broadening of the $511 \mathrm{keV}$ annihilation line. The shape of the $511 \mathrm{keV}$ peak gives thus the one-dimensional momentum distribution $\rho\left(p_{\mathrm{L}}\right)$ of the annihilating electron-positron pairs. A Doppler shift of $1 \mathrm{keV}$ corresponds to a momentum value of $p_{\mathrm{L}}=0.54$ a.u.

The Doppler broadening can be experimentally measured using a Ge gamma ray detector with a good energy resolution. The typical resolution of a detector is around $1-1.5 \mathrm{keV}$ at $500 \mathrm{keV}$. This is considerably compared with the total width of 2-3 keV of the annihilation peak, meaning that the experimental lineshape is strongly influenced by the detector resolution. Therefore, various shape parameters are used to characterize the $511 \mathrm{keV}$ line. The low electron-momentum parameter $S$ is defined as the ratio of the counts in the central region (typically, $p_{\mathrm{L}}<0.4$ a.u.) of the annihilation line to the total number of the counts in the line. In the same way, the high electron-momentum parameter $W$ is the fraction of the counts in the wing regions of the line (typically, $p_{\mathrm{L}}>1.5$ a.u.). Due to their low momenta, valence electrons mainly contribute to the region of the $S$ parameter. On the other hand, only core electrons have momentum values high enough to contribute to the $W$ parameter. Therefore, $S$ and $W$ are sometimes called the valence and core annihilation parameters, respectively.

\section{N-DOPED CVD-ZNO}

Figure 1 shows the $S$ parameter measured at room temperature as a function of positron implantation energy in $\mathrm{N}$-doped CVD-ZnO thin-film samples. When positrons are implanted close to the sample surface with $E=0-1 \mathrm{keV}$, a relatively high $S$ parameter of up to $S=0.50$ is recorded in the samples. These values characterize the defects and chemical nature of the near-surface region of the samples 
at the depth $0-5 \mathrm{~nm}$. The region of constant $S$ gives the characteristic value of the CVD-ZnO layer and extends up to $8 \mathrm{keV}$ in the thickest sample. The decrease of the $S$ parameter at higher implantation energies is due to the increasing fraction of positrons annihilating in the HT-ZnO substrate for which the characteristic $S$ parameter is roughly $S=0.44$, slightly above the value of the "defect-free" $\mathrm{ZnO}$ lattice. ${ }^{11}$

The $S$ and $W$ parameters measured in the N-doped CVD-ZnO layers are shown as plotted against each other with the positron implantation energy as running parameters in Fig. 2. The values characterizing the layers are highlighted in the figure. Figure 2 also shows the values of the $\mathrm{ZnO}$ lattice, ${ }^{11}$ the isolated $\mathrm{Zn}$ vacancy, ${ }^{20,21}$ and those typical of HT-ZnO, ${ }^{22,23}$ adjusted for the detector resolu-

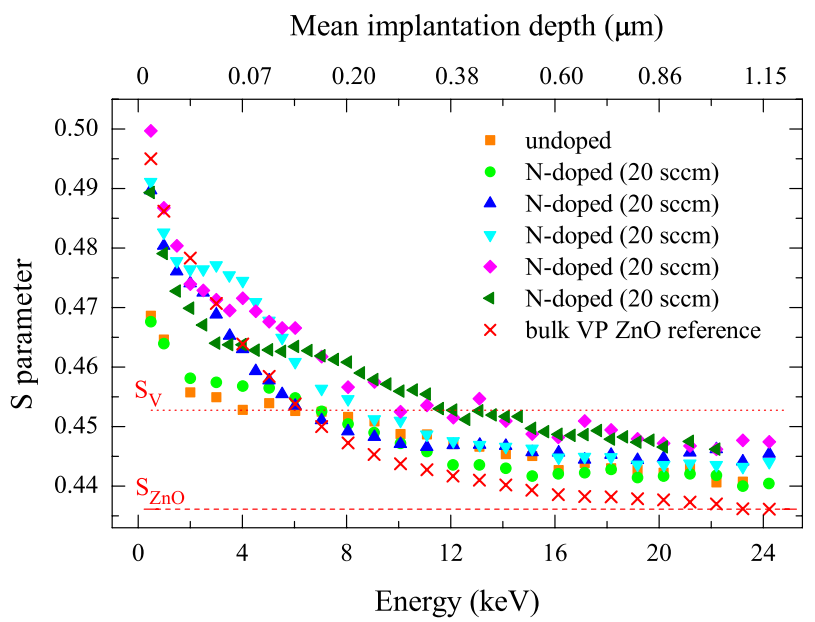

FIG. 1. $S$ parameter as a function of positron implantation energy (probing depth) in CVD-grown $\mathrm{ZnO}: \mathrm{N}$ thin films. The $S$ parameters characterizing the $\mathrm{ZnO}$ lattice and the $\mathrm{Zn}$ vacancy are shown as dashed lines, determined based on the measurement data in the bulk VP ZnO (Ref. 11).

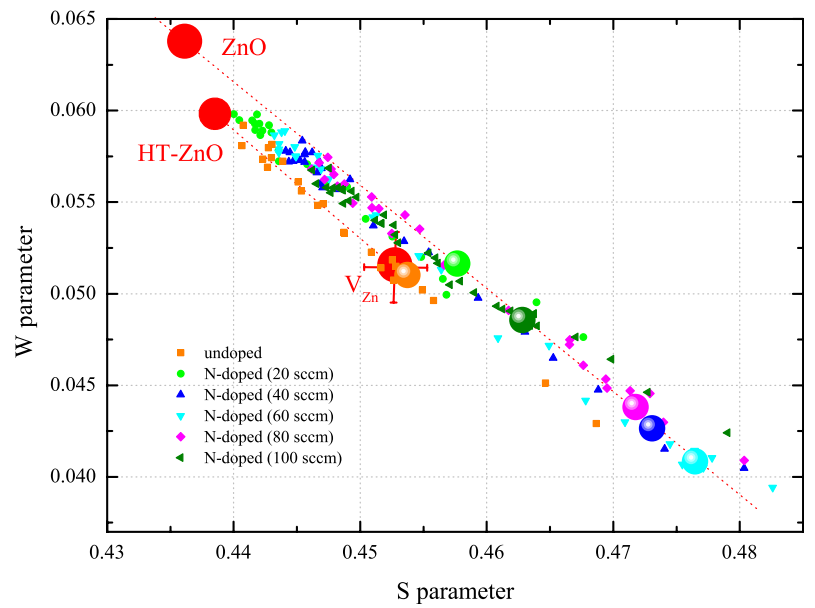

FIG. 2. $(S, W)$ plot with positron implantation energy as the running parameter. The characteristic $(S, W)$ points of the $\mathrm{ZnO}$ lattice, $V_{\mathrm{Zn}}$, and HT-ZnO (essentially $\mathrm{Li}_{\mathrm{Zn}}$ ) are shown for reference. tion, and geometry applied in these experiments. It is clearly seen that at high positron implantation energies, the measured data converge toward the HT-ZnO point as expected. Interestingly, the $(S, W)$ point measured in the undoped CVD-ZnO sample coincides with the $\mathrm{Zn}$ vacancy point. This is interpreted as saturation trapping to single $\mathrm{Zn}$ vacancy-related defects, whose concentration hence needs to be above $5 \times 10^{18}$ per $\mathrm{cm}^{3} .{ }^{21}$ On the other hand, the $(S, W)$ points characterizing the N-doped CVD-ZnO samples all fall on a line that goes through the $\mathrm{ZnO}$ lattice values $\left(S_{\mathrm{B}}, W_{\mathrm{B}}\right)$ and runs to the right of the $\mathrm{ZnO}-V_{\mathrm{Zn}}$ line. This together with the relatively high $S$ parameter values $\left(S\right.$ goes up to $\left.1.09 \times S_{\mathrm{B}}\right)$ indicates that the $\mathrm{N}$-doped samples contain important concentrations of clusters of vacancies, containing both $\mathrm{Zn}$ and $\mathrm{O}$ vacancies (clusters of $\mathrm{Zn}$ vacancies only would be indistinguishable from single $\mathrm{Zn}$ vacancies $\left.{ }^{24}\right)$. Importantly, the linear behavior of the $(S, W)$ parameters in the $\mathrm{N}$-doped samples implies that the vacancy clusters have similar nature in all the samples and only their concentration changes.

Figure 2 shows that the $S$ parameter increases with $\mathrm{N}$ flow up to $60 \mathrm{sccm}$ and then decreases again, indicating that the relationship between the $\mathrm{N}$ content and the concentration of the observed vacancy clusters is not straightforward. To determine the possible effect of negative ions on the room temperature data, we measured the N-doped $\mathrm{ZnO}$ samples at temperatures ranging from 40 to $500 \mathrm{~K}$ (data from a representative sample are shown in Fig. 3). The $S$ parameter measured in the layer decreases with decreasing temperature, indicating that negative ions compete with vacancies in trapping positrons at low temperatures, as the negative ions produce the annihilation parameters of the defect-free lattice. The data are similar for all the $\mathrm{N}$-doped samples; hence the differences in the $S$ parameters measured at room temperature are due to differences in vacancy cluster contents rather than differences in negative ion introduction. It should be noted that the temperature dependence is very similar in $\mathrm{N}$-implanted $\mathrm{HT} \mathrm{ZnO}$; also,

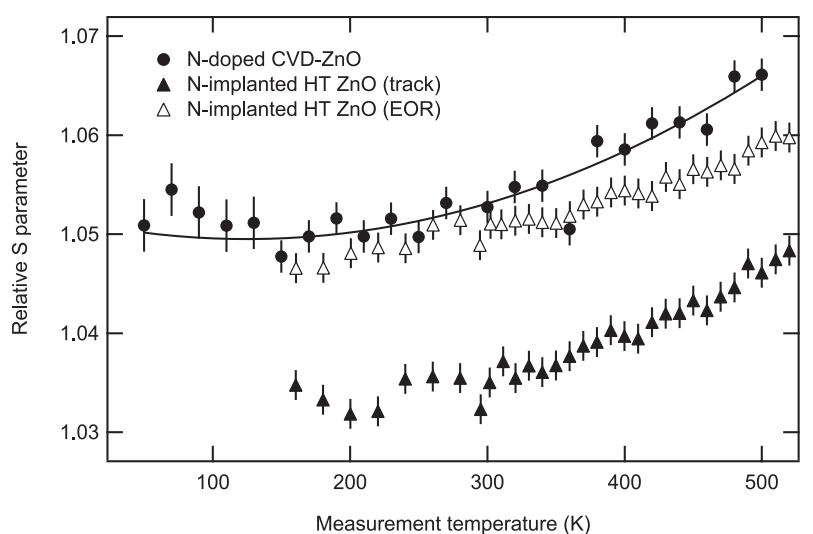

FIG. 3. $S$ parameter as a function of temperature in the $20-\mathrm{sccm} \mathrm{ZnO}: \mathrm{N}$ thin film sample. The solid curve is drawn to guide the eye. Data from $\mathrm{N}$-implanted HT ZnO are shown for comparison. 
vacancy clusters and negative ion-type defects have been observed. ${ }^{4}$

\section{N-DOPED CVT-ZNO}

Figure 4 shows the average positron lifetime measured as a function of temperature in a bulk N-doped CVT-ZnO crystal as well as the second (vacancy) lifetime component extracted from the annihilation spectra. Results also from undoped $\mathrm{CVT}^{25}$ and vapor-phase grown (VP) $\mathrm{ZnO}^{11}$ are shown for comparison. The increase of the average positron lifetime $\tau_{\text {ave }}$ with decreasing temperature is a clear indication of the presence of negatively charged vacancy defects. In undoped $\mathrm{VP} \mathrm{ZnO}$, the monotonous behavior of the lifetime below $300 \mathrm{~K}$ indicates that these vacancy defects are the dominant negatively charged defect. On the other hand, in the undoped CVT ZnO sample, the slight change in the temperature dependence of the positron lifetime at $50-150 \mathrm{~K}$ is a sign of negative ion-type defects competing in trapping of positrons with the negative vacancies. This is observed also as the increase of the first lifetime component $\tau_{1}$ (not shown here) where the increased trapping in the negative ion-type defects brings the component closer to the bulk lifetime.

A longer lifetime component of $\tau_{\mathrm{V}}=\tau_{2}=230 \pm 10 \mathrm{ps}$ can be extracted from the exponential lifetime spectra measured in the undoped CVT and VP samples below $300 \mathrm{~K}$, whereas the data measured in the undoped $\mathrm{ZnO}$ sample above $300 \mathrm{~K}$ exhibit only one component. The longer lifetime component is constant as a function of temperature,

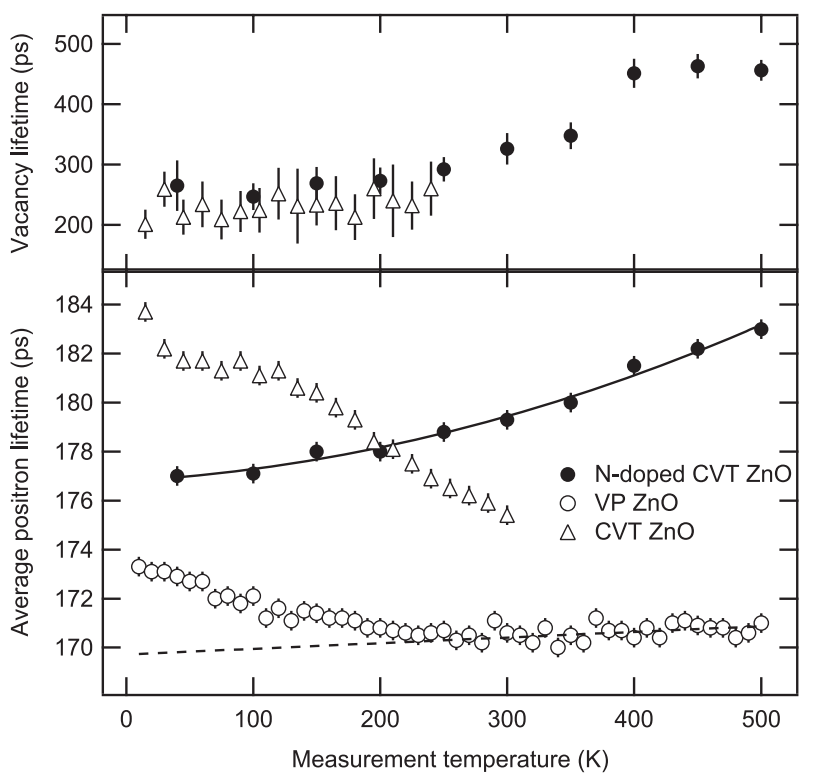

FIG. 4. Average positron lifetime and the longer lifetime component as a function of measurement temperature measured in N-doped CVT $\mathrm{ZnO}$. Data for undoped CVT $\mathrm{ZnO}$ and $\mathrm{VP} \mathrm{ZnO}$ are shown for comparison. The solid curve is drawn to guide the eye, whereas the dashed line describes the effect of thermal expansion of the lattice on the $\mathrm{ZnO}$ lattice lifetime. implying that it represents positrons annihilation in one trapped state. This lifetime component is specific to the $\mathrm{Zn}$ vacancy. The lifetime $\tau_{\mathrm{B}}=170 \mathrm{ps}$ is attributed o the annihilation from the delocalized state in the $\mathrm{ZnO}$ lattice at $300 \mathrm{~K}$, whereas the slight increase of the average positron lifetime with temperature above $300 \mathrm{~K}$ in the undoped VP $\mathrm{ZnO}$ sample is due to thermal expansion. ${ }^{11}$ The $\mathrm{Zn}$ vacancy concentrations in the undoped VP and CVT $\mathrm{ZnO}$ can be estimated to be $2 \times 10^{15}$ per $\mathrm{cm}^{3}$ and $2 \times 10^{16}$ per $\mathrm{cm}^{3}$, respectively. ${ }^{11,25}$

The lifetime data obtained in the $\mathrm{N}$-doped CVT ZnO are less straightforward to interpret. Vacancy defects are clearly present in this material, as the average positron lifetime is above the $\mathrm{ZnO}$ lattice lifetime (dashed line in Fig. 4). On the other hand, the decrease of the average positron lifetime with decreasing temperature is a sign of negative ion-type defects competing with the vacancies in positron trapping. Further, the vacancy lifetime component extracted from the lifetime spectra is quite long, about $450 \mathrm{ps,} \mathrm{in} \mathrm{particular} \mathrm{at} \mathrm{higher}$ temperatures, whereas it decreases to below 300 ps at lower temperatures. The long lifetime of about $450 \mathrm{ps}$ is indicative of the presence of large vacancy clusters where several $\mathrm{Zn}$ and $\mathrm{O}$ atoms are missing. The decrease of the vacancy lifetime component with decreasing temperature suggests that negatively charged $\mathrm{Zn}$ vacancies are present, too, and pull down the vacancy lifetime component when their attractiveness increases thanks to the $T^{-1 / 2}$ dependence of the trapping coefficient. For this effect to be observable, the vacancy clusters need to be neutral. It should be noted that the decrease of the average positron lifetime with decreasing temperature cannot be explained by the $\mathrm{Zn}$ vacancies becoming more attractive, as this effect alone would increase the average positron lifetime.

\section{DISCUSSION}

The results obtained in N-doped thin-film CVD ZnO and $\mathrm{N}$-doped bulk CVT ZnO crystals are very similar in nature: all the samples are found to contain relatively large vacancy clusters and negative ion-type defects, whereas positron data in undoped $\mathrm{ZnO}$ are usually dominated by a signal from defects related to single $\mathrm{Zn}$ vacancies. Earlier results ${ }^{4}$ on $\mathrm{N}$-implantation into $\mathrm{ZnO}$ are also quite similar. Already in the as-implanted samples, vacancy clusters are observed throughout the ion track and the projected range. Distinct evolution of the annihilation parameters is observed after the thermal treatments (furnace for $1 \mathrm{~h}$ ) in the range of $600-1000{ }^{\circ} \mathrm{C}$. After the highest temperature annealing (data in Fig. 3), N-implanted samples show vacancy clusters and negative ion-type defects. Hence, it seems that whenever $\mathrm{N}$ is present at important concentrations in the $\mathrm{ZnO}$ lattice, the material contains vacancy clusters and negative ions. It is natural to assign the latter to substitutional $\mathrm{N}$ impurities while the prevalence of vacancy clusters (instead of single $\mathrm{Zn}$ vacancy-related defects) is intriguing. 
Earlier studies on $\mathrm{ZnO}$ show that defects related to single $\mathrm{Zn}$ vacancies are the main defects observed with positron annihilation spectroscopy. This is seen in both undoped and $\mathrm{Al} / \mathrm{Ga}$-doped thin films ${ }^{21,26-28}$ as well as in studies in bulk crystals. ${ }^{10-12,22,25}$ Clusters of vacancies in $\mathrm{ZnO}$ have been observed mainly in implantation studies ${ }^{4,23,29-38}$ and also after heavy irradiation. ${ }^{21}$ These vacancy clusters have been shown to disappear (become unstable) in thermal treatments at temperatures of about $800^{\circ} \mathrm{C}$, with a single exception: the $\mathrm{N}$-implantation damage is stable up to at least $1000{ }^{\circ} \mathrm{C}$ in $\mathrm{ZnO}$. Most of the implantation processing (with elements such as $\mathrm{H}, \mathrm{He}, \mathrm{Li}, \mathrm{N}$, $\mathrm{O}$, and $\mathrm{Si}$ ) has been performed on $\mathrm{HT} \mathrm{ZnO}$, i.e., material with a high concentration of Li that itself stabilizes the damage as observed in the electron irradiation experiments. ${ }^{10,12}$ Hence, this cannot be the reason for the exceptional behavior of $\mathrm{N}$ implanted $\mathrm{ZnO}$. A possible explanation could be that out of the so far implanted species, $\mathrm{N}$ could be the only one taking the substitutional position on the $\mathrm{O}$ sublattice. However, more work is clearly required to explain the mechanism of efficient implantation damage stabilization by nitrogen.

To illustrate the situation with $\mathrm{N}$ incorporation and the formation of various kinds of clustered vacancy defects in $\mathrm{ZnO}$, data from a wide variety of $\mathrm{ZnO}$ samples are collected in Fig. 5. Samples where the dominant signal comes from $\mathrm{Zn}$ vacancies produce data that would fall on the line drawn between the " $\mathrm{ZnO}$ lattice" and " $V_{\mathrm{Zn}}$ " points in the figure and are not shown. ${ }^{21} \mathrm{ZnO}$ bulk crystals grown by the hydrothermal method typically produce data that are close to the "HT ZnO" point (left to the $\mathrm{ZnO}-V_{\mathrm{Zn}}$ line) due to high concentrations of substitutional $\mathrm{Li}^{22}$ Implantation processing with elements other than $\mathrm{N}$, followed by ther-

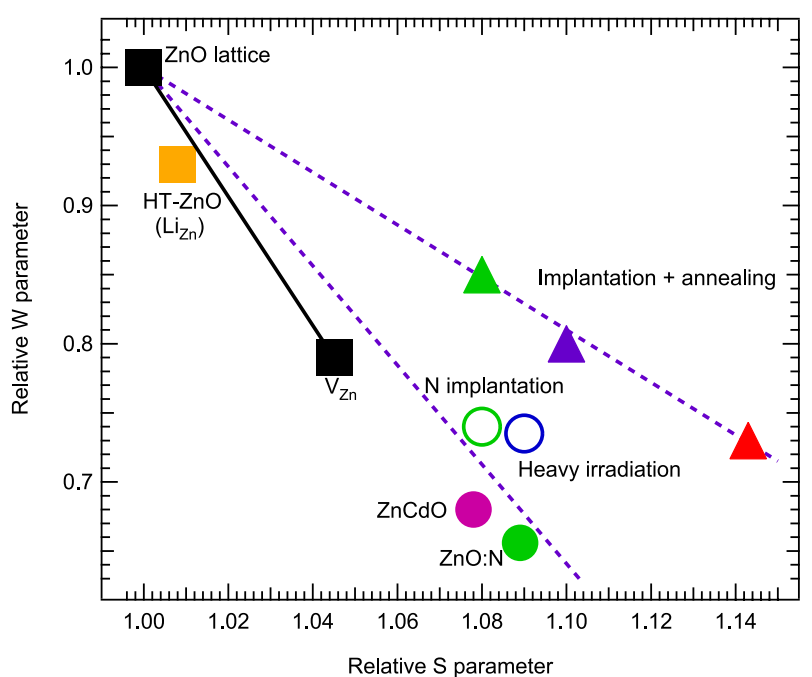

FIG. 5. $(S, W)$ plot with characteristic data from a variety of $\mathrm{ZnO}$ samples. The $S$ and $W$ parameters are shown as normalized to those of the $\mathrm{ZnO}$ lattice. mal treatments (furnace annealing at low temperatures or flash annealing at high temperatures), produce vacancy clusters that are characterized by the triangular symbols and the dashed line going through them and the $\mathrm{ZnO}$ point. These are clearly different in nature from the vacancy clusters introduced during the growth of $\mathrm{N}$-doped $\mathrm{ZnO}$ or, e.g., $\mathrm{ZnCdO},{ }^{39}$ denoted by full circles. $\mathrm{N}$ implantation followed by annealing, ${ }^{4}$ as well as high-fluence particle irradiation, ${ }^{21}$ produce vacancy clusters (open circles) that are rather similar to the in-grown vacancy clusters.

The high stability of the $\mathrm{N}$-implantation induced and in-grown vacancy clusters in $\mathrm{N}$-doped $\mathrm{ZnO}$ - the latter have survived the cooling down from relatively high growth temperatures - correlates with the similar nature observed in positron experiments. On the other hand, the vacancy clusters produced by implantation of other species than $\mathrm{N}$-which disappear during high temperature anneals—are clearly different from the positron point of view. A possible reason is the balance between the amounts of $\mathrm{Zn}$ and $\mathrm{O}$ vacancies forming the clusters in question. A relatively simple idea, based on the positron data trends in another strongly cation-anion mismatched semiconductor $\mathrm{InN},{ }^{24}$ would be that the less stable clusters have more $\mathrm{O}$ vacancies. However, this interpretation is subject to more detailed studies (both experimental and computational) on the nature of multivacancy complexes in $\mathrm{ZnO}$. Finally, an interesting observation that can be made is that in N-containing samples, similar to those presented here, Raman experiments show evidence of interstitial clusters. ${ }^{40}$

\section{SUMMARY}

We have studied $\mathrm{N}$-doped $\mathrm{CVD} \mathrm{ZnO}$ thin films and $\mathrm{N}$-doped CVT bulk $\mathrm{ZnO}$ crystals with positron annihilation spectroscopy, with the aim of elucidating the role of nitrogen in the $\mathrm{ZnO}$ lattice. The positron methods are best suited for studying vacancy-type defects in semiconductors when the defect concentrations are above $10^{15}$ per $\mathrm{cm}^{3}$. A more general discussion of the results is made possible by the comparison with earlier results on a wide variety of undoped, doped, and implantation-processed $\mathrm{ZnO}$ samples.

We show that the introduction of $\mathrm{N}$ impurities into $\mathrm{ZnO}$, irrespective of whether it is done during the growth of thin films or bulk crystals or through implantation and subsequent thermal treatments, leads to the formation of stable vacancy clusters and negative ion-type defects. The latter are quite likely the $\mathrm{N}$ impurities themselves (the concentrations have not been correlated) at substitutional positions. The existence of these vacancy clusters is observed almost exclusively for $\mathrm{N}$ introduction, whereas single $\mathrm{Zn}$ vacancy defects or easily removable vacancy clusters are more typically found in other cases. The close resemblance to effects observed in $\mathrm{ZnO}-\mathrm{CdO}$ alloying suggests that formation of vacancy clusters in $\mathrm{N}$-containing $\mathrm{ZnO}$ could be due to relaxation of stress/strain brought by 
the foreign species rather than electronic (Fermi-level) effects. The relation of the $\mathrm{N}$-induced vacancy clusters with $\mathrm{Zn}$ interstitial complexes needs to be clarified.

\section{ACKNOWLEDGMENTS}

We wish to acknowledge the Academy of Finland for funding this research. Support by the DFG within SFB787 is acknowledged. Partial support was provided by the Department of Energy grant no. DE-FG02-07ER46386 (M.C.T.) and National Science Foundation grant no. DMR-1004804 (M.D.M.).

\section{REFERENCES}

1. B.K. Meyer, H. Alves, D.M. Hofmann, W. Kriegseis, D. Forster, F. Bertram, J. Christen, A. Hoffmann, M. Straßburg, M. Dworzak, U. Haboeck, and A.V. Rodina: Bound exciton and donor-acceptor pair recombinations in ZnO. Phys. Status Solidi B 241, 231 (2004).

2. U. Ozgur, Y.I. Alivov, C. Liu, A. Teke, M.A. Reshchikov, S. Dogan, V. Avrutin, S.J. Cho, and H. Morkoc: A comprehensive review of ZnO materials and devices. J. Appl. Phys. 98, 041301 (2005).

3. C.F. Klingshirn, B.K. Meyer, A. Waag, A. Hoffmann, and J. Geurts: Zinc Oxide. Springer Ser. Mater. Sci. 120, (2010).

4. T.M. Børseth, F. Tuomisto, J.S. Christensen, E.V. Monakhov, B.G. Svensson, and A.Y. Kuznetsov: Vacancy clustering and acceptor activation in nitrogen-implanted ZnO. Phys. Rev. B. 77, 045204 (2008).

5. G. Perillat-Merceroz, F. Donatini, R. Thierry, P-H. Jouneau, P. Ferret, and G. Feuillet: Structural recovery of ion implanted ZnO nanowires. J. Appl. Phys. 111, 083524 (2012).

6. M.A. Myers, M.T. Myers, M.J. General, J.H. Lee, L. Shao, and $\mathrm{H}$. Wang: P-type $\mathrm{ZnO}$ thin films achieved by $\mathrm{N}^{+}$ion implantation through dynamic annealing process. Appl. Phys. Lett. 101, 112101 (2012).

7. S. Lautenschlaeger, S. Eisermann, B.K. Meyer, G. Callison, M.R. Wagner, and A. Hoffmann: Nitrogen incorporation in homoepitaxial ZnO CVD epilayers. Phys. Status Solidi RRL 3, 16 (2009).

8. S. Lautenschlaeger, S. Eisermann, G. Haas, E.A. Zolnowski, M.N. Hofmann, A. Laufer, M. Pinnisch, B.K. Meyer, M.R. Wagner, J.S. Reparaz, G. Callsen, A. Hoffmann, A. Chernikov, S. Chatterjee, V. Bornwasser, and M. Koch: Optical signatures of nitrogen acceptors in ZnO. Phys. Rev. B 85, 235204 (2012).

9. M.C. Tarun, M. Zafar Iqbal, and M.D. McCluskey: Nitrogen is a deep acceptor in ZnO. AIP Adv. 1, 022105 (2011).

10. W. Puff, S. Brunner, P. Mascher, and A.G. Balogh: Studies of defects in electron and proton irradiated $\mathrm{ZnO}$ by positron annihilation. Mater. Sci. Forum 196-201, 333 (1995).

11. F. Tuomisto, V. Ranki, K. Saarinen, and D.C. Look: Evidence of the $\mathrm{Zn}$ vacancy acting as the dominant acceptor in n-type $\mathrm{ZnO}$. Phys. Rev. Lett. 91, 205502 (2003).

12. Z.Q. Chen, S.J. Wang, M. Maekawa, A. Kawasuso, H. Naramoto, X.L. Yuan, and T. Sekiguchi: Thermal evolution of defects in as-grown and electron-irradiated $\mathrm{ZnO}$ studied by positron annihilation. Phys. Rev. B 75, 245206 (2007).

13. S. Lautenschlaeger, J. Sann, N. Volbers, B.K. Meyer, A. Hoffmann, U. Haboeck, and M.R. Wagner: Asymmetry in the excitonic recombinations and impurity incorporation of the two polar faces of homoepitaxially grown ZnO films. Phys. Rev. B 77, 144108 (2008).

14. M.R. Wagner, T.P. Bartel, R. Kirste, A. Hoffmann, J. Sann, S. Lautenschläger, B.K. Meyer, and C. Kisielowski: Influence of substrate surface polarity on homoepitaxial growth of $\mathrm{ZnO}$ layers by chemical vapor deposition. Phys. Rev. B 79, 035307 (2009).
15. S.J. Jokela and M.D. McCluskey: Hydrogen complexes in $\mathrm{ZnO}$ grown by chemical vapor transport. Physica B 401-402, 395 (2007).

16. Cermet, Inc: http://www.cermetinc.com.

17. K. Saarinen, P. Hautojärvi, and C. Corbel: Positron annihilation spectroscopy of defects in semiconductors, in Identification of Defects in Semiconductors (Semiconductors and Semimetals), Vol. 51A, edited by M. Stavola (Academic Press, New York, 1998), p. 209.

18. F. Tuomisto and I. Makkonen: Defect identification in semiconductors: Experiment and theory of positron annihilation. Rev. Mod. Phys, to be published.

19. M.J. Puska and R.M. Nieminen: Theory of positrons in solids and on solid surfaces. Rev. Mod. Phys. 66, 841 (1994).

20. F. Tuomisto, K. Saarinen, D.C. Look, and G.C. Farlow: Introduction and recovery of point defects in electron-irradiated $\mathrm{ZnO}$. Phys. Rev. B 72, 085206 (2005).

21. A. Zubiaga, F. Tuomisto, V.A. Coleman, H.H. Tan, C. Jagadish, K. Koike, S. Sasa, M. Inoue, and M. Yano: Mechanisms of electrical isolation in $\mathrm{O}^{+}$-irradiated $\mathrm{ZnO}$. Phys. Rev. B 78, 035125 (2008).

22. K.M. Johansen, A. Zubiaga, I. Makkonen, F. Tuomisto, P.T. Neuvonen, K.E. Knutsen, E.V. Monakhov, A.Y. Kuznetsov, and B.G. Svensson: Identification of substitutional $\mathrm{Li}$ in n-type $\mathrm{ZnO}$ and its role as an acceptor. Phys. Rev. B 83, 245208 (2011).

23. K.M. Johansen, A. Zubiaga, F. Tuomisto, E.V. Monakhov, A.Y. Kuznetsov, and B.G. Svensson: $\mathrm{H}$ passivation of Li on $\mathrm{Zn}$-site in $\mathrm{ZnO}$ : Positron annihilation spectroscopy and secondary ion mass spectrometry. Phys. Rev. B 84, 115203 (2011).

24. C. Rauch, I. Makkonen, and F. Tuomisto: Identifying vacancy complexes in compound semiconductors with positron annihilation spectroscopy: A case study of InN. Phys. Rev. B 84, 125201 (2011).

25. F. Tuomisto, A. Mycielski, and K. Grasza: Vacancy defects in (Zn, Mn)O. Superlattices Microstruct. 42, 218 (2007).

26. A. Zubiaga, F. Tuomisto, F. Plazaola, K. Saarinen, J.A. Garcia, J.F. Rommeluere, J. Zuñiga-Pérez, and V. Muñoz-San José: Zinc vacancies in the heteroepitaxy of $\mathrm{ZnO}$ on sapphire: Influence of the substrate orientation and layer thickness. Appl. Phys. Lett. 86, 042103 (2005).

27. A. Zubiaga, F. Tuomisto, J. Zuñiga-Pérez, and V. Muñoz-San José: Characterization of non-polar $\mathrm{ZnO}$ layers with positron annihilation spectroscopy. Acta Phys. Pol. A 114, 1457 (2008).

28. D.C. Look, K.D. Leedy, L. Vines, B.G. Svensson, A. Zubiaga, F. Tuomisto, D. Doutt, and L.J. Brillson: Self-compensation in semiconductors: The Zn-vacancy in Ga-doped ZnO. Phys. Rev. B 84, 115202 (2011).

29. Z.Q. Chen, M. Maekawa, S. Yamamoto, A. Kawasuso, X.L. Yuan, T. Sekiguchi, R. Suzuki, and T. Ohdaira: Evolution of voids in $\mathrm{Al}^{+}$-implanted $\mathrm{ZnO}$ probed by a slow positron beam. Phys. Rev. B 69, 035210 (2004).

30. Z.Q. Chen, T. Sekiguchi, X.L. Yuan, M. Maekawa, and A. Kawasuso: $\mathrm{N}^{+}$ion-implantation-induced defects in $\mathrm{ZnO}$ studied with a slow positron beam. J. Phys. Condens. Matter 16, S293 (2004).

31. Z.Q. Chen, A. Kawasuso, Y. Xu, H. Naramoto, X.L. Yuan, T. Sekiguchi, R. Suzuki, and T. Ohdaira: Production and recovery of defects in phosphorus-implanted ZnO. J. Appl. Phys. 97, 013528 (2005).

32. Z.Q. Chen, A. Kawasuso, Y. Xu, H. Naramoto, X.L. Yuan, T. Sekiguchi, R. Suzuki, and T. Ohdaira: Microvoid formation in hydrogen-implanted $\mathrm{ZnO}$ probed by a slow positron beam. Phys. Rev. B 71, 115213 (2005).

33. Z.Q. Chen, M. Maekawa, A. Kawasuso, R. Suzuki, and T. Ohdaira: Interaction of nitrogen with vacancy defects in $\mathrm{N}^{+}$-implanted $\mathrm{ZnO}$ studied using a slow positron beam. Appl. Phys. Lett. 87, 091910 (2005).

34. G. Brauer, W. Anwand, W. Skorupa, J. Kuriplach, O. Melikhova, and C. Moisson: Defects in virgin and $\mathrm{N}^{+}$-implanted $\mathrm{ZnO}$ single 
crystals studied by positron annihilation, Hall effect, and deep-level transient spectroscopy. Phys. Rev. B 74, 045208 (2006).

35. Z.Q. Chen, M. Maekawa, and A. Kawasuso: Energy variable slow positron beam study of $\mathrm{Li}^{+}$-implantation-induced defects in $\mathrm{ZnO}$ Chin. Phys. Lett. 23, 675 (2006).

36. Z.Q. Chen, M. Maekawa, A. Kawasuso, S. Sakai, and H. Naramoto: Annealing process of ion-implantation-induced defects in $\mathrm{ZnO}$ : Chemical effect of the ion species. J. Appl. Phys. 99, 093507 (2006).

37. T.M. Børseth, F. Tuomisto, J.S. Christensen, W. Skorupa, E.V. Monakhov, B.G. Svensson, and A.Y. Kuznetsov: Deactivation of $\mathrm{Li}$ by vacancy clusters in ion-implanted and flash-annealed $\mathrm{ZnO}$. Phys. Rev. B 74, 161202R (2006).

38. P.T. Neuvonen, L. Vines, V. Venkatachalapathy, A. Zubiaga, F. Tuomisto, A. Hallén, B.G. Svensson, and A.Y. Kuznetsov: Defect evolution and impurity migration in Na implanted ZnO. Phys. Rev. B 84, 205202 (2011)

39. A. Zubiaga, F. Plazaola, J.A. García, F. Reurings, F. Tuomisto, A.Y. Kuznetsov, W. Egger, J. Zuñiga-Pérez, and V. Muñoz-Sanjosé: Role of vacancy clusters in $\mathrm{Zn}_{1-\mathrm{x}} \mathrm{Cd}_{\mathrm{x}} \mathrm{O}$ alloys. J. Appl. Phys. 113, 032512 (2013).

40. N.H. Nickel: Unpublished. 